\title{
Energy and Time Efficient Broadcasting in Known Topology Radio Networks
}

\author{
Leszek Gasieniec $^{1 \star}$, Erez Kantor ${ }^{2 \star \star}$, Dariusz R. Kowalski ${ }^{1 \star}$, \\ David Peleg ${ }^{2 \star \star}$, and Chang $\mathrm{Su}^{1}$ \\ 1 Department of Computer Science, \\ The University of Liverpool, Liverpool L69 3BX, UK \\ \{leszek, darek, suc\}@csc.liv.ac.uk \\ 2 Department of Computer Science and Applied Mathematics, \\ The Weizmann Institute of Science, Rehovot, 76100 Israel \\ \{erez.kantor, david.peleg\}@weizmann.ac.il
}

\begin{abstract}
The paper considers broadcasting protocols in radio networks with known topology that are efficient in both time and energy. The radio network is modelled as an undirected graph $G=(V, E)$ where $|V|=n$. It is assumed that during execution of the communication task every node in $V$ is allowed to transmit at most once. Under this assumption it is shown that any radio broadcast protocol requires $D+\Omega(\sqrt{n-D})$ transmission rounds, where $D$ is the diameter of $G$. This lower bound is complemented with an efficient construction of a deterministic protocol that accomplishes broadcasting in $D+O(\sqrt{n} \log n)$ rounds. Moreover, if we allow each node to transmit at most $k$ times, the lower bound $D+\Omega\left((n-D)^{1 /(2 k)}\right)$ on the number of transmission rounds holds. We also provide a randomised protocol that accomplishes broadcasting in $D+O\left(k n^{1 /(k-2)} \log ^{2} n\right)$ rounds. The paper concludes with a discussion of several other strategies for energy efficient radio broadcasting and a number of open problems in the area.
\end{abstract}

\section{Introduction}

\subsection{Background}

This paper concerns the study of simultaneously energy and time efficient communication protocols under an abstract model of radio networks, where uniform transmitting and receiving devices form a set of nodes $V$ in an undirected graph $G=(V, E)$ of size $|V|=n$. Two nodes $v, w \in V$ are neighbours in $G$, i.e., there is an edge $(v, w)$ in $E$, whenever $v$ and $w$ can communicate (i.e., send and receive messages) directly with each other. Nodes that are not connected by edges must communicate via intermediate nodes. We consider synchronous networks, where the processing and transmission speeds of nodes are uniform across the entire

\footnotetext{
* Supported in part by the Royal Society grant Algorithmic and Combinatorial Aspects of Radio Communication, IJP - 2006/R2.

** Supported in part by grants from the Minerva Foundation and the Israel Ministry of Science.
} 
network. Communication is performed in rounds. During any round, each node can be either in a transmitting mode or in a receiving mode, meaning that a node cannot both transmit and receive messages during the same round. Moreover, if a node $v$ transmits in a given round, the message is delivered to all its neighbours. However, a node $w$ in a receiving mode in a given round will receive the message from its transmitting neighbour $v$ if and only if $v$ is the only transmitting neighbour of $w$ in this round. The efficiency of communication protocols in synchronous networks is often expressed as the time (the number of rounds) required to accomplish the task. In this paper, apart from the time complexity we are also interested in another important efficiency measure, namely the energy efficiency. We consider strategies for efficient radio communication in the context of the broadcasting task.

In the broadcasting problem, a distinguished node $s$ in the network, referred to as the source node, has a message that has to be distributed to all other nodes in the network. Energy efficient radio broadcasting was mostly studied in the context of geometric networks, where the network nodes are embedded into 2-dimensional plane. In particular, the goal in the energy efficient broadcast tree problem is to find a transmission graph that minimizes the total power consumption and contains a directed spanning tree rooted at the source node $s$. The problem is known to be NP-hard [8] and if the distance function is arbitrary, it has no logarithmic factor approximation unless $P=N P$ [16]. For the Euclidean distance model, Wan et al. [22] and with improved reasoning Klasing et al. [17] argued that the algorithm that computes a minimal spanning tree for the set of nodes yields the approximation ratio 12.15 . The approximation ratio was further reduced to 7.6 by Flammini et al. [10] and later to 6.33 by Navarra [21]. Recently Ambühl [1] showed that the minimum spanning tree yields an approximation ratio 6 , which is as far as one can go with this approach, in view of the lower bound presented in [22].

In our model of radio communication spatial information is not available, thus there is a need for an alternative definition of energy consumption. Since the network nodes are uniform, it is natural to assume that transmissions performed by every node cost exactly the same. Moreover, we are interested in balancing the energy consumption at the nodes. This would serve to avoid energy consumption bottlenecks at some wireless nodes, especially if they operate on limited power sources, e.g., batteries, and may thus help to prolong the operational lifetime of the entire system. With this goal in mind, we consider energy efficient strategies in which every node is allowed to transmit at most once during the execution of any communication task, in our case the broadcasting procedure. We refer to such energy efficient strategies as 1-shot protocols. Such a strategy for energy efficient radio communication was very recently studied in the context of broadcasting and gossiping in radio networks of random topology, see [5].

Research on time efficient broadcasting in known topology radio networks, where an entire schedule of node transmissions can be precomputed in advance, was initiated in [6]. In this paper Chlamtac and Weinstein provided a broadcast schedule with the running time $O\left(D \log ^{2} n\right)$, where $D$ is diameter of the network. 
Later an $\Omega\left(\log ^{2} n\right)$ time lower bound was proved for the family of radius 2 graphs [3]. While it was known for quite a while [4] that for every $n$-vertex radio network of diameter $D$ there exists a deterministic broadcasting schedule of length $O\left(D \log n+\log ^{2} n\right)$, an appropriate efficient construction for such a schedule was proposed only very recently in [18]. Another type of a broadcast schedule requiring $D+O\left(\log ^{5} n\right)$ rounds is due to Gaber and Mansour, see [11]. Elkin and Kortsarz in [9] presented deterministic constructions of broadcasting schedules of length $D+O\left(\log ^{4} n\right)$ for arbitrary graphs and $D+O\left(\log ^{3} n\right)$ for planar graphs. The existential proof that the optimal $D+O\left(\log ^{2} n\right)$ broadcast schedule is feasible was given by Gasieniec et al. in [12]. Explicit constructions of broadcasting schedules operating in $O\left(D+\log ^{2} n\right)$ and $D+O\left(\frac{\log ^{3} n}{\log \log n}\right)$ rounds can be found in [19] and [7] respectively.

\subsection{Our results}

In this paper we focus on simultaneously time and energy efficient broadcasting protocols in radio networks with known topology. In Section 2 we show that any 1-shot radio broadcast protocol requires $D+\Omega(\sqrt{n-D})$ rounds of transmission, where $D$ is the diameter of $G$. In Section 3 we provide an efficient construction of a deterministic protocol that accomplishes broadcasting in $D+O(\sqrt{n} \log n)$ rounds. Section 4 contains results in the model where each node is allowed to transmit at most $k$ times. We prove a lower bound $D+\Omega\left((n-D)^{1 /(2 k)}\right)$ and we design a randomised protocol that accomplishes broadcasting in $D+O\left(k n^{1 /(k-2)} \log ^{2} n\right)$ rounds. Finally in Section 5 we discuss several other strategies for energy efficient radio broadcasting and state a number of open problems in the area. All algorithms presented in the paper are deterministic and constructible in polynomial time.

\section{A $D+\Omega(\sqrt{n-D})$ lower bound for 1-shot broadcasting}

In this section we show that there exist radio networks in which every 1-shot broadcasting strategy requires at least $D+\Omega(\sqrt{n-D})$ rounds of transmissions. Specifically, we show that for any positive integer $n$ there exists an $n$-node bipartite graph on which any 1-shot broadcasting protocol requires $\Omega(\sqrt{n})$ communication rounds. Consider the binomial graph $B(x)=(\{r\} \cup U \cup L, E)$, where $\{r\}, U$ and $L$ are disjoint, $|U|=x,|L|=y$, and $y=\left(\begin{array}{l}x \\ 2\end{array}\right)$. The singleton set $\{r\}$ is intended as the source while the set $U$ (respectively, $L$ ) forms the upper (resp., lower) layer of $B(x)$. The nodes in $U$ are labelled by the integers 1 to $x$ and the nodes in $L$ by unordered pairs $\{a, b\}$ such that $1 \leq a<b \leq x$. The node $r$ is connected to all the nodes in $U$, and a node in $L$ labelled by $\{a, b\}$ is connected to exactly two nodes $a$ and $b$ in $U$. For example, see structures of $B(3)$ and $B(4)$ in Figures $1(\mathrm{a})$ and 1(b) respectively. In the first step, the message is transmitted by $r$ to reach all the nodes in $U$. Our analysis concerns the process by which the message is disseminated from the nodes of $U$ to the nodes of $L$.

The proof is based on the observation that in any 1-shot broadcasting protocol, exactly one node from the upper layer $U$ is permitted to transmit in each round. Consider the first round of any broadcasting procedure. Assume first that 
two nodes $a$ and $b$ in $U$ decide to transmit simultaneously. Their shared neighbour $\{a, b\}$ in the lower layer $L$ gets neither of the messages due to collision. Moreover, this node will not receive any other messages in the future since its only neighbours already transmitted and they are allowed to do this only once. This proves that multiple transmissions in the first round are not allowed. Now assume that during the first round a single node $a$ from $U$ transmits and all neighbours of $a$ in $L$ receive the broadcast message. Removing $a$ from $U$ and all its neighbours from $L$, we obtain a smaller binomial graph $B(x-1)$. Thus the next round of the broadcasting protocol must again consist of a transmission from a single node in $U$. This argument is repeated until a binomial graph $B(1)$ with no edges between the layers $U$ and $L$ is obtained. This leads to the conclusion that any 1-shot broadcasting protocol in a binomial graph $B(x)$ requires $x-1$ consecutive transmission rounds. Since $n=x+y+1$ for $y=\left(\begin{array}{l}x \\ 2\end{array}\right)$, the number of communication rounds required by any 1-shot broadcasting procedure is $\Omega(\sqrt{n})$.

Theorem 1. There exists a radio network of size $n$ and diameter $D$ in which any 1-shot broadcasting protocol requires $D+\Omega(\sqrt{n-D})$ transmission rounds.

Proof. The lower bound of $D+\Omega(\sqrt{n-D})$ can be derived directly from the lower bound for 1-shot broadcasting strategies in binomial bipartite graphs. Consider a graph formed by attaching a path $P$ of length $D-2$ to the node $r$ of the binomial bipartite graph $B(x)$, where $x$ is the largest integer satisfying $D-2+$ $x(x+1) / 2 \leq n$, and placing the broadcasting source at the far (from $B(x)$ ) end of the path $P$. The time required by any 1 -shot broadcasting procedure includes $D-2$ rounds to move the broadcast message along $P$ and $\Omega(\sqrt{n-(D-2)})$ additional rounds to inform every node in the lower layer of $B(x)$. The lower bound $D-2+\Omega(\sqrt{n-(D-2)})=D+\Omega(\sqrt{n-D})$ follows.

\section{1-shot broadcasting in $D+O(\sqrt{n} \log n)$ rounds}

For ease of presentation, we first provide a 1-shot radio broadcasting strategy for all $n$-node bipartite graphs (with the message already available at all nodes of the upper layer), consisting of $O(\sqrt{n})$ transmission rounds. This result is then combined with the new ranking scheme given in Section 3.2, and admits 1-shot radio broadcasting in $D+O(\sqrt{n} \log n)$ rounds for arbitrary undirected graphs.

\subsection{Broadcasting in bipartite graphs}

Consider a bipartite graph $B=(U \cup L, E)$ with the upper layer $U$ and the lower layer $L$. In what follows we assume that $U$ forms a minimal covering set (MCS) of $L$, see [13], i.e., that the removal of any node from $U$, along with all edges incident to it, isolates some nodes in $L$. Define another graph $G^{\prime}=\left(U, E^{\prime}\right)$ on the basis of $B$ as follows. For every node $v \in L$ of degree at least two, pick two arbitrary neighbours of $v$ in $U$, and add to $E^{\prime}$ an edge between them. Note that 


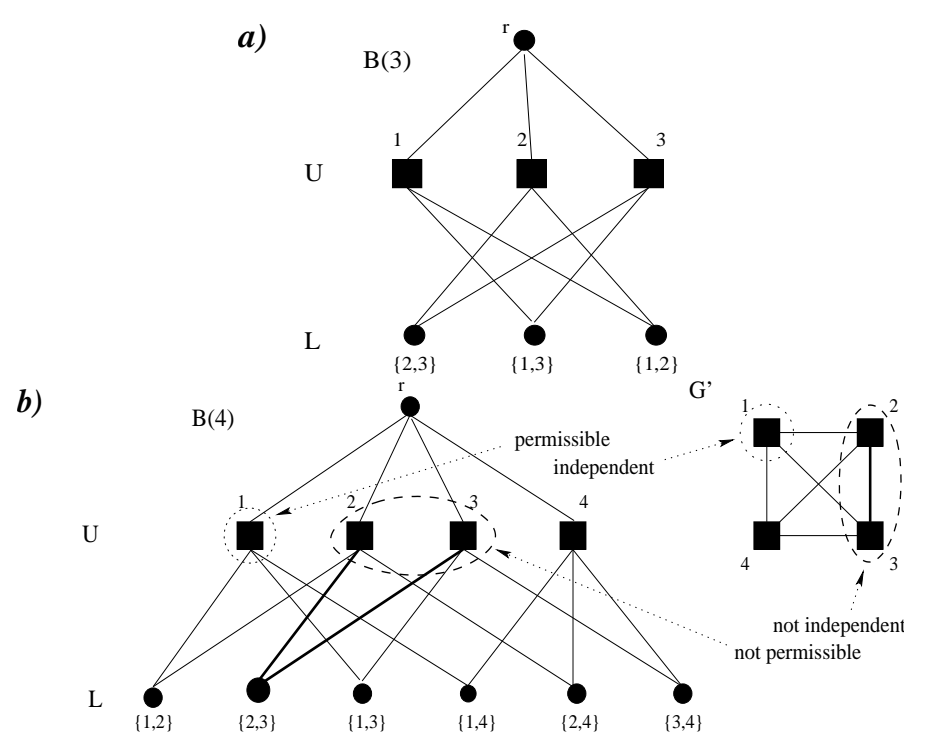

Fig. 1. (a) the structure of $B(3) ; \quad$ (b) the graph $G^{\prime}$ based on $B(4)$.

by this construction, $\left|E^{\prime}\right| \leq|L|$. For example, a graph $G^{\prime}$ defined on the basis of the layers $U$ and $L$ in the binomial graph $B(4)$ is presented in Figure 1(b).

A set of nodes in an undirected graph is independent if no two nodes in it are directly connected by an edge. For a subset $Q \subseteq U$, let $I(Q) \subseteq L$ denote the set containing all nodes in $L$ having exactly one neighbour in $Q$. We say that a subset $Q \subseteq U$ in the bipartite graph $B$ is permissible if simultaneous transmissions from $Q$ inform a subset $I(Q) \subseteq L$ and the removal of all nodes in $Q$ from $U$, along with their incident edges, does not isolate nodes in $L \backslash I(Q)$.

Lemma 1. An independent set of nodes $Q$ in the graph $G^{\prime}$ forms a permissible set in $B$.

Proof. By the definition of $I(Q)$, it suffices to show that removing all nodes in $Q$ from the upper layer $U$, along with their incident edges, does not isolate any nodes in $L \backslash I(Q)$. Indeed, consider an arbitrary node $v \in L$ with neighbour set $A$ in $B$. If $v$ has no neighbour in $Q$, then after the removal, $v$ is still connected to some node in $U \backslash Q$. If $v$ has exactly one neighbour in $Q$, then $v \in I(Q)$, hence $v \notin L \backslash I(Q)$. Finally, suppose $v$ is connected to at least two nodes in $Q$. In this case, $v$ must also have another neighbour outside of $Q$. To see this, note that by construction, the graph $G^{\prime}$ includes an edge between some two nodes of $A$. Hence if $A \subseteq Q$, then $Q$ fails to be independent in $G^{\prime}$, leading to contradiction.

The following fact, related to the efficient construction of large independent sets, was proved in the context of parallel computing in [14]. 
Lemma 2. In a graph $G^{\prime}=\left(U, E^{\prime}\right)$, where $|U|=x$ and $\left|E^{\prime}\right|=y$, one can construct in time polynomial in $x+y$ an independent set $Q \subseteq U$ such that $|Q| \geq \frac{x^{2}}{2 y+x}$.

Combining Lemmas 1 and 2 we conclude:

Lemma 3. In a bipartite graph $B(U \cup L, E)$, where $|U|=x,|L|=y$ and $x \geq \sqrt{y}$, there exists a permissible transmission set of size at least $\left\lceil\frac{x^{2}}{2 y+x}\right\rceil$.

We are ready to state the main theorem for 1-shot radio broadcasting strategies in bipartite graphs.

Theorem 2. In any n-node bipartite graph, the broadcast message can be distributed from the upper layer to the lower layer in $O(\sqrt{n})$ rounds using a 1-shot broadcasting strategy.

Proof. Assume that $U^{\prime} \subseteq U$ is a subset of nodes in the upper layer that forms a minimal covering set of the lower layer $L$, where $\left|U^{\prime}\right|=x$ and $|L|=y$. Note that if $x^{2} \leq 2 y+x$ then nodes from $U^{\prime}$ can transmit sequentially in time $x=O(\sqrt{n})$ since $x^{2} \leq 2 y+x<2(y+x)=2 n$. Otherwise, assume that $x^{2}>2 y+x$. We first show how to reduce the size of the upper layer to $\sqrt{y} \log y$ in $O(\sqrt{n})$ rounds, and later how to perform further reductions to obtain a set of size $\sqrt{y}$ in $O(\sqrt{y})$ additional rounds. After the reduction process is accomplished, the nodes still present in the upper layer transmit sequentially in at most $\sqrt{y}=O(\sqrt{n})$ rounds.

Assume first that after some number of rounds of the reduction process the size of the upper layer $x^{\prime}$ is still larger than $\sqrt{y} \log y$, where the size of the lower layer is $y^{\prime} \leq y$. By Lemma 3, the current set $U^{\prime}$ (after the removal of nodes and edges in the construction process) contains a permissible transmission set of size at least $\frac{\left(x^{\prime}\right)^{2}}{2 y^{\prime}+x^{\prime}}$. We can assume that $x^{\prime} \leq y^{\prime}$ (the upper layer constitutes a minimal covering set) and consequently that there exists a permissible transmission set of size at least $\frac{\left(x^{\prime}\right)^{2}}{3 y^{\prime}}$. In this case, after one round of transmissions the size of the upper layer is reduced to $x^{\prime \prime}=x^{\prime}-\frac{\left(x^{\prime}\right)^{2}}{3 y^{\prime}}=x^{\prime}\left(1-\frac{x^{\prime}}{3 y^{\prime}}\right)$. Since we assumed $x^{\prime}>\sqrt{y} \log y$ and also $y^{\prime} \leq y$, it follows that $x^{\prime \prime} \leq x^{\prime}\left(1-\frac{\sqrt{y} \log y}{3 y}\right)=x^{\prime}\left(1-\frac{\log y}{3 \sqrt{y}}\right)$. This means that the size of the upper layer can be reduced in one round by a fraction of $1-\frac{\log y}{3 \sqrt{y}}$ and consequently in at most $\frac{3 \sqrt{y}}{\log y}$ rounds by a constant fraction, for as long as $x^{\prime}>\sqrt{y} \log y$. Thus repeating this reduction process for $O(\log y)$ times, the size of the upper layer becomes smaller than $\sqrt{y} \log y$. The total number of rounds in the entire reduction process is $O(\sqrt{y})$, where $\sqrt{y}=O(\sqrt{n})$.

After the size of the upper layer is reduced to $\sqrt{y} \log y$, the remaining reduction process is split into $0 \leq i \leq \log \log y$ stages, where during stage $i$, the size $x^{\prime}$ of the upper layer $U^{\prime}$ is reduced from at most $\frac{\sqrt{y} \log y}{2^{i}}$ to at most $\frac{\sqrt{y} \log y}{2^{i+1}}$. Consider an arbitrary stage $i$, where $y^{\prime} \geq x^{\prime}$ is the size of the lower layer. By Lemma $3, U^{\prime}$ contains a permissible transmission set of size at least $\frac{x^{\prime 2}}{2 y^{\prime}+x^{\prime}}$. Since $x^{\prime} \leq y^{\prime} \leq y$ and $x^{\prime} \geq \frac{\sqrt{y} \log y}{2^{i+1}}$ we conclude that there exists a 
permissible transmission set of size $\frac{x^{\prime 2}}{3 y^{\prime}} \geq\left(\frac{\sqrt{y} \log y}{2^{i+1}}\right)^{2} / 3 y \geq \frac{\log ^{2} y}{2^{2(i+2)}}$. To move from stage $i$ to stage $i+1$ one has to remove from the upper layer $U^{\prime}$ at most $\frac{\sqrt{y} \log y}{2^{i+1}}$ nodes. Since during each round we know how to remove at least $\frac{\log ^{2} y}{2^{2(i+2)}}$ nodes, the number of rounds required to move to stage $i+1$ is bounded by $\frac{\sqrt{y} \log y}{2^{i+1}} / \frac{\log ^{2} y}{2^{2(i+2)}}=\frac{2^{i+3} \sqrt{y}}{\log y}$. Thus the total number of rounds of all $\log \log y$ stages is bounded by $\sum_{i=0}^{\log \log y} \frac{2^{i+3} \sqrt{y}}{\log y}=\frac{8 \sqrt{y}}{\log y} \cdot \sum_{i=0}^{\log \log y} 2^{i}=O(\sqrt{y})$, and $\sqrt{y}=O(\sqrt{n})$.

Finally, when the size of the upper layer $U^{\prime}$ becomes smaller than $\sqrt{n}$, the remaining nodes accomplish broadcasting via sequential transmissions.

\subsection{Broadcasting in arbitrary graphs}

In this section we introduce a new tree ranking scheme that enables 1-shot broadcasting protocols in an arbitrary $n$-node graph $G=(V, E)$ of diameter $D$, in $D+O(\sqrt{n} \log n)$ rounds.

The most time-efficient radio broadcasting algorithms in known graphs use the concept of tree ranking, see e.g., $[12,19]$, where the ranks are computed for especially designed BFS spanning tree rooted in the source node $s$. The algorithms use two types of transmissions. Fast transmissions are performed along paths in the tree containing nodes with the same rank. Slow transmissions are designed to move instances of the broadcast message between nodes with different ranks at neighbouring BFS levels. In this type of radio broadcasting algorithms, most nodes are involved in transmissions of both types. Note, however, that in the setting used in this paper, each node is allowed to transmit at most once, which means that the concepts of fast and slow transmissions have to be unified. We therefore propose a new ranking scheme in which the rank of a node corresponds to the unique number of a round when the node transmits. The new rank is a combination of two types of ranks, external and internal.

External ranks The external rank of every node in the network is computed on the basis of the ranking mechanism proposed in the context of gatheringbroadcasting BFS spanning trees [12]. The nodes in the spanning tree get ranks according to a simple principle. All leaves are assigned the rank 1 and each internal node calculates its rank by looking at the maximum rank among its children. If the maximum rank $m$ occurs in only one child, then the rank of the parent is also set to $m$; otherwise, the parent gets the rank $m+1$. It is known that the rank of the root $s$ in a gathering-broadcasting spanning tree of size $n$ is at $\operatorname{most} \log n$. The spanning tree constructed in [12] has also an important property that at any BFS level $d$, if two nodes $v$ and $w$ as well as their respective (disjoint) parents $p(v)$ and $p(w)$ share the same rank, then there are no edges in the network $G$ between nodes $p(v)$ and $w$ as well as between $p(w)$ and $v$. This property allows simultaneous (collision free) transmissions from the two parents towards their children. Note that nodes having the same rank in the spanning tree form a collection of disjoined paths leading towards the root of the tree. We refer to these paths as chains. The bottom end (from the root) of each path is called a tail of the chain. Note that some chains can contain only singleton nodes. 
For example, all leaves are tails in their chains. Now, if the rank (as defined in [12]) of a node is $l$, then the node is assigned the external rank $l_{e x}=2 l-1$ if it is the tail of some chain; otherwise, it gets the external rank $2 l$. Thus the external rank of the root $s$ in the spanning tree is at most $2 \log n$. We refer to new shorter paths based on external ranks as channels.

Internal ranks The system of internal ranks is computed on the basis of external ranks and the broadcasting scheme for bipartite graphs provided in Section 3.1. Let $d(v)$ be the distance between a node $v$ and the root $s$. Network nodes with the same distance $d(\cdot)$ form BFS layers in $G$. Assume that all nodes in the network obtained the external rank from the range $1, \ldots, 2 \log n$. The set of network nodes $V$ is partitioned into channels, where $P_{i, j}$ denotes $j^{\text {th }}$ channel containing nodes with rank $i$. Each channel $P_{i, j}$ constitutes a supernode in the upper layer of the internal rank bipartite graph $B_{I R}(i)$, for $i=1, \ldots, 2 \log n$. The bottom layer of each $B_{I R}(i)$ is formed of all nodes in the network. A supernode $P_{i, j}$ is connected to a node $w$ in the lower layer of $B_{I R}(i)$ if there exists a node $v \in P_{i, j}$ such that $(v, w) \in E, d(w)-d(v)=1$, and $l_{e x}(v)>l_{e x}(w)$. Note that each graph $B_{I R}(i)$, for $i=1, \ldots, 2 \log n$, has at most $2 n$ vertices, since there are at most $n$ supernodes (they are disjoint subsets of nodes from graph $G$ ) and at most $n$ nodes in the lower layer of graph $B_{I R}(i)$. In each $B_{I R}(i)$ we apply the broadcasting scheme from Section 3.1, which allocates to each supernode $P_{i, j}$ in the upper layer a unique number $k$ (of a transmission round) from the range $1, \ldots, f(n)=O(\sqrt{2 n})=O(\sqrt{n})$. In fact, the number $k$ defines the internal rank $l_{i n}(v)$ for any node $v$ present in the supernode $P_{i, j}$. The examples of internal and external ranking can be found in Figure 2.

Combined ranking scheme The new ranking scheme provides a rank to each node $v \in V$ based on its BFS layer $d(v)$, as well as its internal and external ranks, $l_{i n}(v)$ and $l_{e x}(v)$. More precisely, for any node $v \in V$, where $0 \leq d(v) \leq$ $D, 1 \leq l_{e x}(v) \leq 2 \log n$ and $0 \leq l_{\text {in }}(v)<f(n)=O(\sqrt{n})$, define the delay factor of $v$ as $\delta(v)=2 \log n-l_{e x}(v)$. The new rank of the node $v$ is then

$$
l(v)=d(v)+3\left[\delta(v) f(n)+l_{\text {in }}(v)\right] .
$$

Essentially, the new combined rank $l(v)$ corresponds to the transmission time of the node $v$. In other words, in the new broadcasting scheme the node $v$ transmits only once, in round $l(v)$. This means, e.g., that the running time of the new broadcasting scheme is trivially bounded by $D+O(\sqrt{n} \log n)$. What is left to be shown is that the broadcasting scheme works correctly, i.e., that each network node receives the broadcast message on time (before its transmission round).

The intuition behind the definition of $l(v)$ is as follows. The summand $d(v)$ is necessary since in any case, node $v$ cannot receive the source message faster than its distance from the source. Then, node $v$ may need to wait for some number of rounds in order to avoid collisions, which is expressed by the second summand. The reason for the factor 3 is that in order to avoid collisions between the transmissions of $v$ and those of nodes at distance 2 from it, we allow only nodes of distances $3,6,9 \ldots$ from node $v$ to transmit simultaneously with it. To avoid collisions with nodes on the same BFS layer but with a different external 

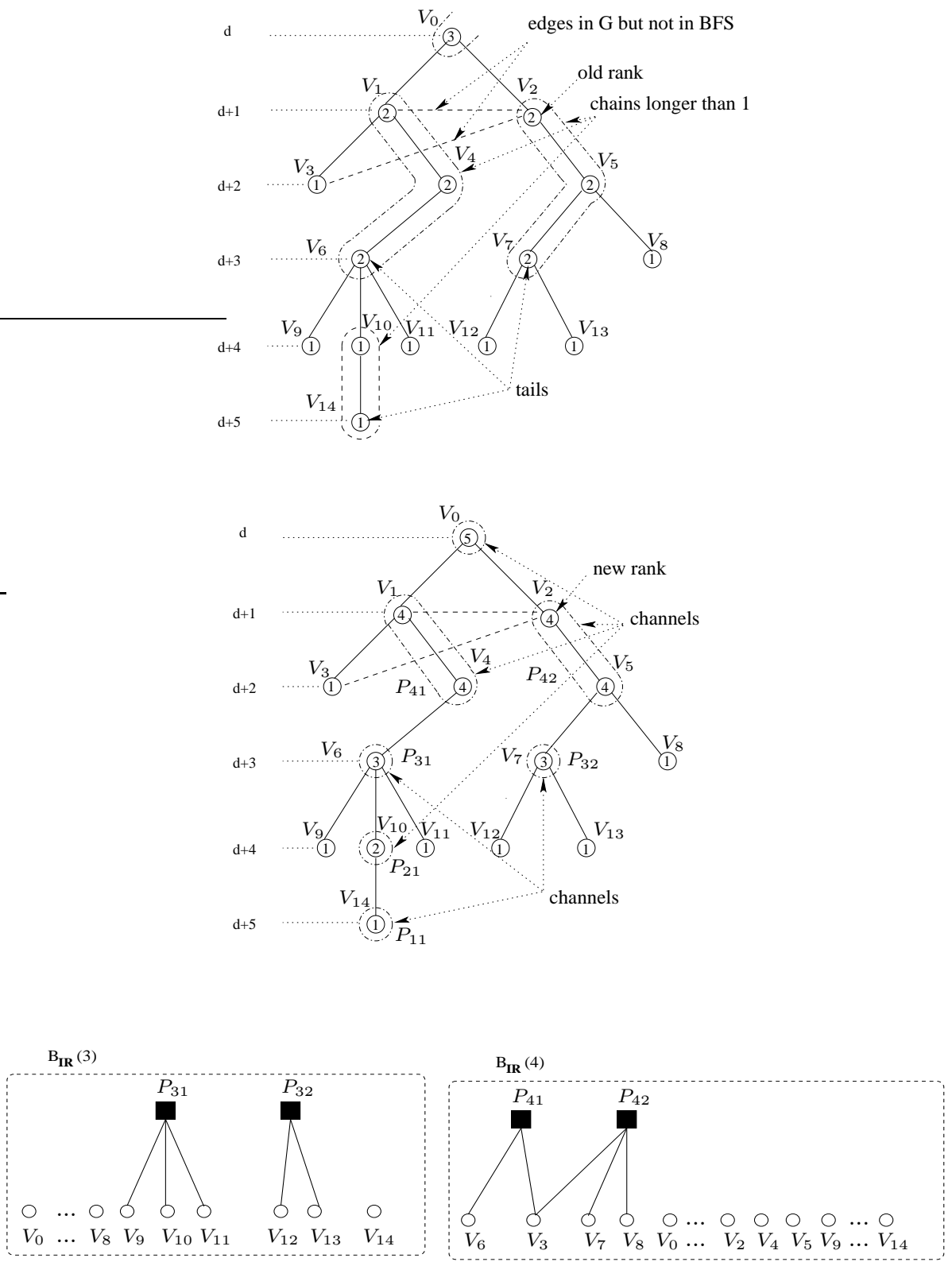

Fig. 2. The old ranks, new external ranks and bipartite graphs $B_{I R}(3)$ and $B_{I R}(4)$

ranking, node $v$ waits for $\delta(v)$ "time windows" of $f(n)$ rounds each. Having done this, only nodes with the same external rank as $v$ may interrupt its transmissions, 
which is dealt with by waiting an additional $l_{i n}(v)$ rounds. The formal analysis of correctness of the algorithm follows.

The proof of the following lemma is deferred to the full version of the paper.

Lemma 4. Each network node $v$ with $0 \leq d(v) \leq D, 1 \leq l_{e x}(v) \leq 2 \log n$ and $0 \leq l_{\text {in }}(v)<f(n)=O(\sqrt{n})$ receives the broadcast message prior to the round $l(v)=d(v)+3\left[\delta(v) f(n)+l_{\text {in }}(v)\right]$.

The following theorem holds.

Theorem 3. In every radio network of size $n$ and a diameter $D$ there exists a 1-shot broadcasting protocol that runs in $D+O(\sqrt{n} \log n)$ rounds

Proof. Recall that each node $v$ is scheduled to transmit only once during time $l(v)=d(v)+3\left[\delta(v) f(n)+l_{i n}(v)\right]$, where $0 \leq d(v) \leq D, \delta(v)=2 \log n-l_{e x}(v), 1 \leq$ $l_{e x}(v) \leq 2 \log n$ and $0 \leq l_{\text {in }}(v)<f(n)=O(\sqrt{n})$. Lemma 4 ensures that each node receives the broadcast message before its transmission time. The time complexity follows directly from the new ranking scheme, where the running time is bounded by the ranking of nodes at the BFS level farthest from the source node $s$, thus it is not more than $D+O(\sqrt{n} \log n)$.

\section{$4 k$-shot protocols}

A natural extension of 1-shot strategies is a model in which each node in the network can transmit at most $k$ times. We show here that under this assumption, both deterministic and randomised radio broadcasting requires $\Omega\left(n^{1 /(2 k)}\right)$ transmission rounds in bipartite graphs, and that this lower bound can be nearly matched by a randomised algorithm. These results could also be generalised for networks with a diameter $D$, in the same fashion as in Sections 2 and 3.2, resulting in the lower bound $D+\Omega\left(n^{1 /(2 k)}\right)$ and the upper bound $D+O\left(k n^{1 /(k-2)} \log n\right)$. Please note that due to the space limit almost all proofs in this section are deferred to the full version of the paper.

\subsection{Lower bound}

The lower bound argument mimics the proof for 1-shot protocols.

Theorem 4. There exist bipartite graphs of size $n$ in which any $k$-shot broadcasting scheme requires $\Omega\left(n^{1 /(2 k)}\right)$ transmission rounds.

Using the same argument as in the proof of Theorem 1 we come to the following conclusion.

Corollary 1. There exist bipartite graphs of size $n$ and diameter $D$ in which any $k$-shot broadcasting scheme requires $D+\Omega\left((n-D)^{1 /(2 k)}\right)$ rounds.

Moreover, it follows that in order to guarantee fast broadcast in bipartite graphs, namely, in $O$ (polylog $n$ ) rounds (or in general graphs in $D+O$ (polylog $n$ ) rounds), an $\Omega\left(\frac{\log n}{\log \log n}\right)$-shot protocol must be used, since $n^{\frac{\log \log n}{2 \log n}}=\sqrt{\log n}$. The same bounds hold also for randomised protocols. 


\subsection{Randomised $k$-shot protocol}

Consider a graph $G=(U, L, E)$. Assume that $n \geq 8$ (otherwise there exists a direct constant length schedule) and $5 \leq k<\frac{\log n}{5+\log \log n}+2$. Note that for $k \leq 4$ one can use efficient deterministic 1-shot protocol described in section 3 . And for $k \geq \frac{\log n}{5+\log \log n}+2$ the broadcast protocol proposed in this section runs in at most $O\left(\log ^{3} n / \log \log n\right)$ rounds, i.e., almost matches the lower bound $\Omega\left(\log ^{2} n\right)$ for unbounded energy broadcast, see [3]. We say that an event holds with high probability if the probability is at least $1-n^{-c / k}$, for some constant $c \geq 1$.

We compute first an MCS for $|U| \leq n / 2 \leq|L|$ and then we define a $k$-shot protocol RAndBroAdcast $(k)$ as follows. Let $a=k-2$. Note that $3 \leq a<$ $\frac{\log n}{5+\log \log n}$. The protocol proceeds in $a$ epochs, where each epoch (apart from the first one) is formed of $\mathcal{T}=64 n^{1 / a} \log n$ consecutive rounds. The first epoch is different and it is executed in $(a+1) \cdot \mathcal{T}$ rounds. We define also a sequence of numbers $\left\langle p_{i}\right\rangle$, for $2 \leq i \leq a$, where

$$
p_{i}=\frac{16 \log n}{n^{(i-1) / a}} \quad \text { and } \quad p_{i}^{\prime}= \begin{cases}p_{2}, \quad i=2, \\ \frac{p_{i}}{\prod_{j=2}^{i-1}\left(1-p_{j}\right)}, & \text { otherwise } .\end{cases}
$$

Applying the inequality $a<\frac{\log n}{5+\log \log n}$ we get $p_{i}<1 / 2$, for every $2 \leq i \leq$ $a$, and consequently $p_{i}<\left(p_{2}\right)^{i-1}<(1 / 2)^{i-1}<\prod_{j=1}^{i}\left(1-p_{j}\right)$. Therefore the following fact holds.

Fact $50<p_{i} \leq p_{i}^{\prime} \leq 1$ for every $2 \leq i \leq a$.

A pseudocode of the k-shot protocol for a node $v \in U$ is presented below.

\section{Algorithm RANDBROADCAST $(k)$}

1. Epoch 1:

- Select, one by one, uniformly at random (with repetitions allowed) $a+1$ integers from the set $\{1,2, \ldots,(a+1) \cdot \mathcal{T}\}$; Let $\alpha_{1}(v)$ be the first selected integer.

- During the period $\{1,2, \ldots,(a+1) \cdot \mathcal{T}\}$ transmit in rounds with the index corresponding to selected integers.

2. A random bit selection:

- Select a random bit $\beta_{2}(v)$, set to 1 with probability $p_{2}^{\prime}$ and to 0 otherwise.

- For $i=3$ to $a$ do:

(i) If $\beta_{j}(v)=0$ for every $2 \leq j \leq i-1$, then select a random bit $\beta_{i}(v)$, set to 1 with probability $p_{i}^{\prime}$ and to 0 otherwise.

3. For $i=2$ to $a$ do:

$/ /$ iterating epochs $i=2, \ldots, a$

If $\beta_{i}(v)=1$ then

- select uniformly at random an integer $1 \leq \alpha_{i}(v) \leq \mathcal{T}$, and

- transmit in round $(a+i-1) \mathcal{T}+\alpha_{i}(v)$. 
Lemma 5. For every $v \in U$,

1. $\beta_{i}(v)=1$ with probability $p_{i}$, for every $2 \leq i \leq a$; and

2. $\sum_{j=2}^{a} \beta_{j}(v) \leq 1$ with probability 1 .

Note that every node $v$ transmits at most once throughout epochs $i=2, \ldots, a$, and in epoch 1 it performs at most $a+1$ additional transmissions. Hence obtain the following corollary.

Corollary 2. RANDBROADCAST $(k)$ is a $k$-shot protocol with probability 1.

The algorithm runs in time $O(k)$ at each node. Thus the total number of transmission rounds is bounded by $(a+1) \cdot \mathcal{T}+(a-1) \cdot \mathcal{T}=O\left(k n^{1 /(k-2)} \log n\right)$.

We prove here that the $\mathrm{k}$-shot protocol performs radio broadcasting with high probability. We start with two technical observations referring to placing balls in bins. Given $x$ balls and $y$ bins, consider a process in which each ball is placed uniformly and independently in a random bin. This process is used to model random selection of transmitting rounds, where nodes (balls) choose their transmission rounds (bins) randomly. We say that an event is good when more than $x / 2$ bins are occupied, i.e., which where more than $x / 2$ different rounds are selected by a node. Let $\mathcal{P}$ be the probability of a good event. Note that a good event admits existence of a round selected by exactly one node among all considered subset of nodes. (This is due to the fact that at least one bin is occupied by exactly one ball).

Lemma 6. $\mathcal{P} \geq 1-\sum_{j=1}^{\lfloor x / 2\rfloor}\left(\frac{e \cdot(x / 2)}{y}\right)^{x-j}$.

Proof. Let $j$ be the number of occupied bins. We have

$$
\begin{aligned}
\mathcal{P} & =1-\sum_{j=1}^{\lfloor x / 2\rfloor}\left(\begin{array}{l}
y \\
j
\end{array}\right)\left(\frac{j}{y}\right)^{x} \geq 1-\sum_{j=1}^{\lfloor x / 2\rfloor}\left(\frac{e \cdot y}{j}\right)^{j} \cdot\left(\frac{j}{y}\right)^{x}=1-\sum_{j=1}^{\lfloor x / 2\rfloor} e^{j} \cdot\left(\frac{j}{y}\right)^{x-j} \\
& \geq 1-\sum_{j=1}^{\lfloor x / 2\rfloor}\left(\frac{e \cdot(x / 2)}{y}\right)^{x-j} .
\end{aligned}
$$

Lemma 7. If $8 \log n \leq x \leq 3 \mathcal{T} / 8$ and $y \geq \mathcal{T}$, then with probability at least $1-3 / n^{3}$ there exists a bin with exactly one ball.

Proof. It is sufficient to prove that $\mathcal{P} \geq 1-1 / n^{3}$, since if the number of occupied bins is larger than $x / 2$ then there is a bin containing exactly one ball. Using Lemma 6 for $x$ balls and $y=\mathcal{T}$ bins,

$$
\begin{aligned}
\mathcal{P} & \geq 1-\sum_{j=1}^{\lfloor x / 2\rfloor}\left(\frac{e \cdot(x / 2)}{y}\right)^{x-j} \geq 1-\sum_{\ell=\lceil x / 2\rceil}^{x-1}\left(\frac{3 e \mathcal{T} / 16}{\mathcal{T}}\right)^{\ell} \\
& \geq 1-\left(\frac{3 e}{16}\right)^{x / 2} \cdot \frac{1}{1-\frac{3 e}{16}} \geq 1-3 \cdot\left(\frac{3 e}{16}\right)^{(8 \log n) / 2} \geq 1-3 / n^{3} .
\end{aligned}
$$


Lemma 8. Every node $w \in L$ gets the source message with high probability.

Therefore the following theorem holds.

Theorem 6. Algorithm RANDBROADCAST $(k)$ is a $k$-shot protocol and it accomplishes radio broadcasting in $O\left(k n^{1 /(k-2)} \log n\right)$ rounds with high probability.

Note that the ranking scheme proposed in Section 3.2 can be used in conjunction with $k$-shot protocols. I.e., any $O(f(n))$-time $k$-shot broadcasting scheme for bipartite graphs admits $D+O(f(n) \log n)$ broadcast in arbitrary graphs with the diameter $D$. In particular, the $k$-shot randomised protocol $\operatorname{RANDBROADCAST}(k)$ for bipartite graphs can be extended in polynomial time into a protocol for completing broadcast on an arbitrary $D$-hop radio network in $D+O\left(k n^{1 /(k-2)} \log ^{2} n\right)$ rounds, with high probability. More precisely, given a arbitrary graph $G(V, E)$ with diameter $D$, we construct bipartite graphs $B_{I R}(i)$ as in section 3.2, and for each node $v$ we compute $k$ internal ranks $l_{i n}(1, v), l_{i n}(2, v), \ldots, l_{i n}(k, v)$, instead of one internal rank $l_{\text {in }}(v)$, using RandBroadcast $(k)$ algorithm in place of 1 -shot deterministic algorithm. Note that $l_{i n}(j, v)$, for $1 \leq j \leq k$, are now from the range $1, \ldots, f(k, n)$, where $f(n, k)=O\left(k n^{1 /(k-2)} \log n\right)$ due to theorem 6 . Thus combining the concept of external ranks with newly obtained $k$ different internal ranks (there are at most $k$ different rounds in which node $v$ transmits) in the same fashion as in formula (1) in section 3.2 we get the following corollary.

Corollary 3. There exists a polynomial time constructable $k$-shot randomised broadcasting protocol that runs in $D+O\left(k n^{1 /(k-2)} \log ^{2} n\right)$ rounds in every radio network of size $n$ and a diameter $D$, with high probability.

\section{$5 \quad$ Further discussion}

This paper presents a new broadcasting scheme that performs the communication task under assumption that each node can transmit at most once. It turns out that there is a clear distinction between the model with bounded and unbounded number of transmissions. In the unbounded model the lower bound and the upper bound is known to be $D+\Theta\left(\log ^{2} n\right)$ in view of [3] and [12] while in the model with unique transmissions the lower bound is $D+\Omega(\sqrt{n-D})$. Note also that our new 1-shot broadcasting scheme requires $D+O(\sqrt{n} \log n)$ rounds of communication. This leaves an interesting open problem on the exact complexity of 1-shot broadcasting strategies in radio networks with known topology.

Broadcasting with bounded number of transmissions at each node. In the more general case where each node can transmit up to $k$ times during a broadcasting process, it is shown that radio broadcasting requires $\Omega\left(n^{1 /(2 k)}\right)$ transmission rounds in bipartite graphs, and $D+\Omega\left((n-D)^{1 /(2 k)}\right)$ in graphs of diameter $D$. These complexities can be nearly matched by randomised algorithms, however the exact complexity of $k$-shot broadcasting in bipartite and arbitrary radio networks with known topology remains open (the gap is nearly $n^{1 / k}$ ). In particular, a randomised $k$-shot broadcasting algorithm needs $D+O\left(k n^{1 /(k-2)} \log ^{2} n\right)$ 
rounds to succeed in any $D$-hop network, with high probability. Constructing, in polynomial time, an efficient $k$-shot deterministic protocol is another problem that remains open.

Broadcasting with the minimum number of transmissions Another possible strategy for energy efficient radio broadcasting is to minimise the total number of transmissions, without targeting the best possible running time of a broadcasting procedure. In this model one can provide almost immediately an approximate solution based on the efficient computation of minimal connected dominating sets with logarithmic approximation ratio in general graphs [15] and constant approximation ratio in unit disk graphs, see e.g. [2]. An interesting related open problem is to look for trade-offs between the total number of transmissions and the broadcasting time.

\section{Acknowledgments}

We would like to thank Andrzej Lingas for valuable discussions on algorithmic issues related to the main themes of this paper.

\section{References}

1. C. Ambühl. An optimal bound for the MST algorithm to compute energy efficient broadcast trees in wireless networks. Proc. 32nd Int. Coll. on Automata, Languages and Programming (ICALP), 2005, pp. 1139-1150.

2. C. Ambühl, T. Erlebach, M. Mihalak, and M. Nunkesser. Constant-factor approximation for minimum-weight (connected) dominating sets in unit disk graphs. Proceedings of 9th International Workshop on Approximation Algorithms for Combinatorial Optimisation Problems (APPROX), 2006, pp. 3-14.

3. N. Alon, A. Bar-Noy, N. Linial, and D. Peleg. A lower bound for radio broadcast. J. Computer and System Sciences 43 (1991) pp. 290-298.

4. R. Bar-Yehuda, O. Goldreich, and A. Itai. On the time complexity of broadcasting in radio networks: an exponential gap between determinism and randomization. Proc. 5th Symposium on Principles of Distributed Computing (PODC), 1986, pp. 98-107.

5. P. Berenbrink, C. Cooper, and Z. Hu. Energy efficient randomised communication in unknown adhoc networks. Proc. 19th ACM Symposium on Parallelism in Algorithms and Architectures (SPAA), 2007, pp. 250-259.

6. I. Chlamtac and O. Weinstein. The wave expansion approach to broadcasting in multihop radio networks. IEEE Trans. on Communications 39, (1991) pp. 426-433.

7. F. Cicalese, F. Manne, and Q. Xin. Faster centralised communication in radio networks. Proc. 17th International Symposium on Algorithms and Computation (ISAAC), 2006, pp. 339-348.

8. A.E.F. Clementi, P. Crescenzi, P. Penna, G. Rossi, and P. Vocca. On the complexity of computing minimum energy consumption broadcast subgraphs. Proc. 18th Annual Symposium on Theoretical Aspects of Computer Science (STACS), 2001, pp. 121-131.

9. M. Elkin and G. Kortsarz. Improved broadcast schedule for radio networks. Proc. 16th ACM-SIAM Symposium on Discrete Algorithms (SODA), 2005, pp. 222-231. 
10. M. Flammini, A. Navarra, R. Klasing, and S. Perennes. Improved approximation results for the minimum energy broadcasting problem. Proc. DIALM-POMC Workshop on Foundations of Mobile Computing, 2004, pp. 85-91.

11. I. Gaber and Y. Mansour. Centralised broadcast in multihop radio networks. $J$. Algorithms 46(1) (2003) pp. 1-20.

12. L. Gasieniec, D. Peleg, and Q. Xin. Faster communication in known topology radio networks. Proc. 24th Annual ACM Symposium on Principles of Distributed Computing (PODC), 2005, pp. 129-137.

13. L. Gasieniec, I. Potapov, and Q. Xin. Efficient gossiping in known radio networks. Proc. 11th Int. Colloq. on Structural Information and Communication Complexity (SIROCCO), 2004, LNCS 3104, pp. 173-184.

14. M. Goldberg and T. Spencer. An efficient parallel algorithm that finds independent sets of guaranteed size. SIAM J. of Discrete Mathematics, Vol. 6, No. 3 (1993) pp. 443-459.

15. S. Guha and S. Khuller. Approximation algorithms for connected dominating sets. Algorithmica, Vol. 20, No. 4 (1998) pp. 374-387.

16. S. Guha and S. Khuller. Improved methods for approximating node-weighted Steiner trees and connected dominating sets. Information and Computation, Vol. 150 (1999) pp. 57-74.

17. R. Klasing, A. Navarra, A. Papadopoulos, and S. Perennes. Adaptive broadcast consumption (abc), a new heuristic and new bounds for the minimum energy broadcast routing problem. Networking, (2004) pp. 866-877.

18. D.R. Kowalski and A. Pelc. Centralised deterministic broadcasting in undirected multi-hop radio networks. Proc. 7th International Workshop on Approximation Algorithms for Combinatorial Optimisation Problems (APPROX), 2004, pp. 171182.

19. D.R. Kowalski and A. Pelc. Optimal deterministic broadcasting in known topology radio networks. Distributed Computing, 19(3) (2007) pp. 185-195.

20. M. Mitzenmacher and E. Upfal. "Probability and Computing," Cambridge University Press, 2005.

21. A. Navarra. Tighter bounds for the minimum energy broadcasting problem. Proc. 3rd International Symposium on Modeling and Optimisation in Mobile, Ad-Hoc and Wireless Networks, 2005, pp. 313-322.

22. P.J. Wan, G. Calinescu, X.Y. Li, and O. Frieder. Minimum-energy broadcast routing in static ad hoc wireless networks. Proc. 20th Annual Joint Conference of the IEEE Computer and Communications Societies (INFOCOM), 2001, pp. 1162-1171. 